\title{
Transcatheter Versus Surgical Aortic Valve Replacement in Renal Transplant Patients: A Meta-Analysis
}

\author{
Tanveer Mir ${ }^{\mathrm{a}}$, Fahed Darmoch ${ }^{\mathrm{b}}$, Waqas Ullah ${ }^{\mathrm{c}}$, Yasar Sattar ${ }^{\mathrm{d}}$, Zaher Hakime, \\ Homam Moussa Pacha ${ }^{\mathrm{f}}$, Lina Fouadg, Delair Gardie, James J. Glaziere, \\ Kenton Zehre, M. Chadi Alraies ${ }^{\mathrm{e}, \mathrm{h}}$
}

\begin{abstract}
Background: The outcome of transcutaneous aortic valve replacement (TAVR) in patients with kidney transplant is unknown, as majority of these patients were excluded from the major TAVR clinical trials. We sought to compare patients with severe aortic stenosis who underwent TAVR versus surgical aortic valve replacement (SAVR) with a history of kidney transplant.
\end{abstract}

Methods: PubMed, Google Scholar and Cochrane databases were searched to identify relevant articles. The incidence of all-cause mortality and acute kidney injury (AKI) was calculated using relative risk on a random effect model.

Results: A total of 1,538 patients (TAVR 328, SAVR 1,210) were included in the study. TAVR was associated with lower mortality as compared with SAVR at 30 days from the index procedure (odds ratio (OR) $0.48,95 \%$ confidence interval (CI): $0.25-0.93$; $\mathrm{P}=0.03$ ). Oneyear mortality was studied in three studies and showed comparable mortality in patients undergoing TAVR and SAVR (OR: 0.76, 95\% CI: 0.10 - 5.51; $\mathrm{P}=0.78)$. Compared to SAVR, TAVR carries an identical risk of AKI (OR: 0.44, 95\% CI: $0.10-1.90$; P = 0.27). A sensitivity analysis performed by exclusion of Voudris et al study showed a non-significant difference in the mortality incidence of two groups at 30 days (OR: $0.72,95 \%$ CI: $0.27-1.91 ; \mathrm{P}=0.51$ ).

Manuscript submitted May 19, 2020, accepted June 10, 2020

Published online August 1, 2020

${ }^{a}$ Wayne State University/Detroit Medical Center, Detroit, MI, USA

bInternal Medicine, Beth Israel Deaconess Medical Center/Harvard School of Medicine, Boston, Massachusetts, MA, USA

'Internal Medicine, Abington Jefferson Health, 1200 Old York Road, Abington, PA 19044, USA

dnternal Medicine, Icahn School of Medicine at Mount Sinai Elmhurst Hospital, New York, NY, USA

${ }^{e}$ Cardiovascular Medicine, Detroit Medical Center, Wayne State University, Detroit, MI, USA

fUniversity of Texas Health Science Center, McGovern Medical School, Memorial Hermann Heart \& Vascular Institute, Houston, TX, USA

gDetroit Medical Center, Heart Hospital, Detroit, MI, USA

${ }^{\mathrm{h}}$ Corresponding Author: M. Chadi Alraies, Wayne State University/Detroit Medical Center, 311 Mack Ave, Detroit, MI 48201, USA.

Email: alraies@hotmail.com

doi: https://doi.org/10.14740/cr1092
Conclusions: In patients with a history of kidney transplant, TAVR was associated with a comparable risk of mortality and AKI compared to SAVR.

Keywords: TAVR; SAVR; Aortic stenosis; Renal transplant

\section{Introduction}

Cardiovascular disease is considered the leading cause of death in patients with end-stage renal disease (ESRD) on chronic dialysis $[1,2]$. Aortic stenosis (AS) is the most common valvular pathology in this population with AS being an independent risk factor for death in these patients. Patients with ESRD have rapid progression of AS, valvular and paravalvular, due to severe calcification and hemodynamic changes during hemodialysis and predispose to endocarditis [3-6]. Chronic kidney disease (CKD) stage $>3$ is an important predictor of outcome and worsens 30-day mortality, acute kidney injury (AKI), and stroke for both surgical aortic valve replacement (SAVR) and transcatheter aortic valve replacement (TAVR) $[7,8]$.

Chronic hemodialysis patient with AS were routinely referred for SAVR. However, SAVR has been associated with higher mortality and adverse outcomes $[9,10]$. Therefore, TAVR has emerged as an alternative treatment option for patients with severe AS who are low, intermediate and high surgical risk candidates [11-13]. However, the outcome of TAVR in patients with ESRD and recipients of kidney transplant is not well known since these patients were excluded from major TAVR clinical trials [11].

The outcome of ESRD patients who undergo TAVR is worse than those who undergo the procedure with normal kidney function [14]. Furthermore, there is a debate on whether SAVR or TAVR is comparable, and which procedure is better in terms of mortality outcomes in kidney transplant patients. Few observational data suggest comparable outcome of TAVR and SAVR in ESRD patients who are chronically on hemodialysis [15-16].

Given this limited TAVR and SAVR outcome data in patients with ESRD on hemodialysis who received kidney transplant, we sought to study the patient characteristics, procedural outcomes and hospital length of stay of TAVR 
compared with SAVR in recipients of kidney transplant with severe AS.

\section{Materials and Methods}

As for the methods, we searched Medline, EMBASE and Cochrane library for randomized controlled trial (RCT) studies that compared SAVR and TAVR outcomes in kidney transplant patients. The combinations of medical terms "aortic stenosis", "valve replacement", "TAVR", "SAVR", and "outcome" were employed to conduct a comprehensive search in the above-mentioned databases. All searches were restricted to studies conducted in human subjects published from the date of the databases' inception through November 2019. There was no language restriction or use of additional filters. A cross-reference check of previously published reviews and/or meta-analyses on this topic was performed. The literature searches and all analyses were conducted following the Preferred Reporting Items for Systematic Reviews and Meta-Analyses (PRISMA) guidelines and the PRISMA statement for network meta-analyses. After initial search and exclusion of duplicate studies and studies with no clear comparison between TAVR and SAVR in kidney transplant, a total of 10 studies were identified for the meta-analysis. The second selection step involved proof-reading of those articles to ensure that the first step was performed correctly. Articles were excluded if data on SAVR or TAVR for kidney transplant was missing or primary and secondary outcomes of interest were not available. In case of unclarity, inclusion of the studies was discussed amongst the authors to arrive at a final decision. A total of four studies were selected finally for comparison of outcome for TAVR versus SAVR in kidney transplant (Fig. 1).

The primary objective of the study is to evaluate the 30 day and 1-year mortality, AKI, hospital stay in days of TAVR procedure compared with SAVR procedure in patients with kidney transplant. As limited trials are available, we collected all available data which compares the two variables in kidney transplant patients for better understanding of outcome in terms of bigger sample size.

\section{Data extraction and analysis}

After careful title checking and reviewing full texts of all the studies, two investigators independently verified the inclusion criteria and abstracted the data from all the papers that met the inclusion criteria. Disagreements were resolved by consensus. All extracted data from the included studies were collected into a spreadsheet and verified by a third author. Summarized and weighted means and rates from each individual trial or observation study for baseline characteristics were reported. Data were pooled for the primary and secondary outcomes using summary risk ratios (RRs) and 95\% confidence interval (CI) using random effects models, while the within- and between-study variance was taken into account. Revman 5.3 was used for meta-analysis of the study. Two-sided P values

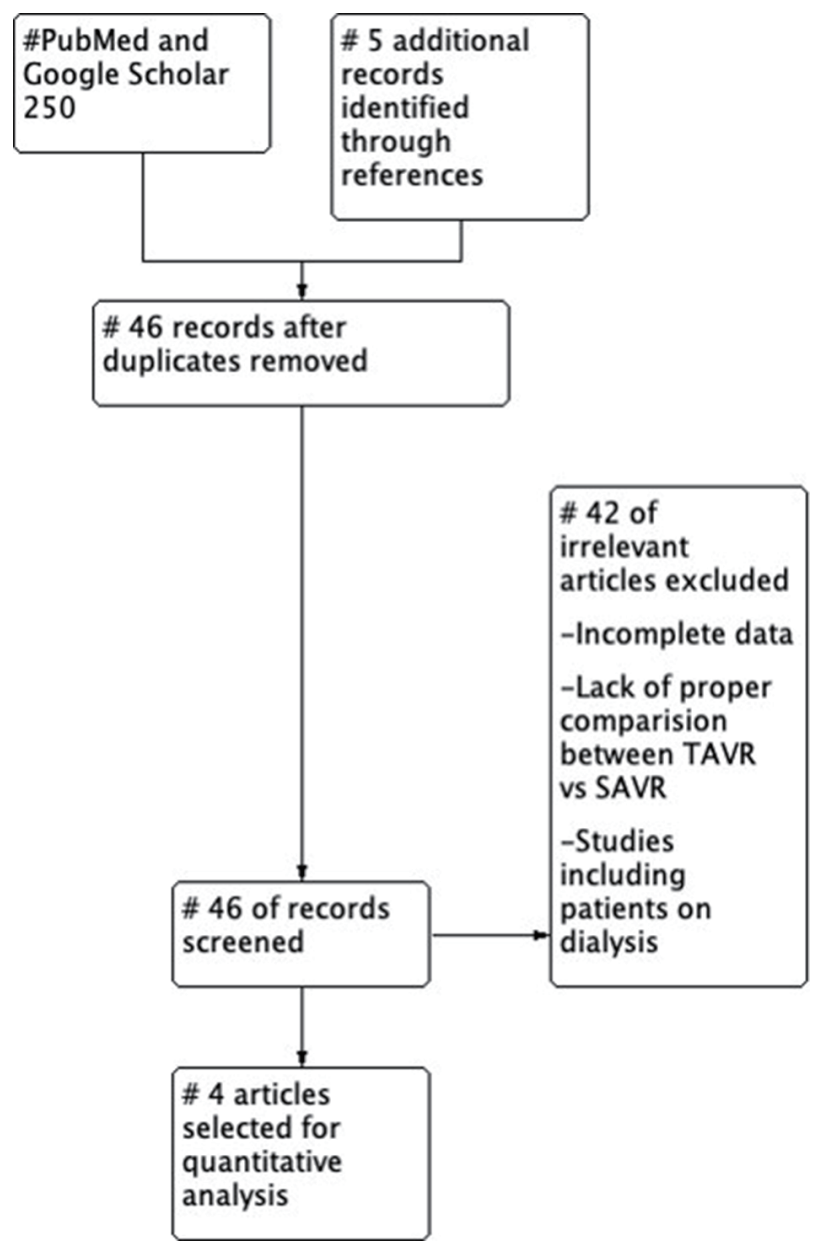

Figure 1. PRISMA flow chart for selection of studies. PRISMA: Preferred Reporting Items for Systematic Reviews and Meta-Analyses.

were calculated with $\mathrm{P}<0.05$ being considered significant for all tests. Heterogeneity was assessed by means of the I-square statistic (with an I-square value $>75 \%$ being considered the result of severe heterogeneity).

Sensitivity analysis was performed for different study design and baseline characteristics to evaluate for the consistency of the main results across all studies that were included in the analysis. A two-tailed $\mathrm{P}$ value of $<0.05$ was considered statistically significant. All data and supporting materials have been provided with the published article and all supporting data are available within the article.

\section{End-point outcomes}

Four studies were included in the meta-analysis from 250 identified studies. All studies included in this study are retrospective studies. Studies were performed between 2013 and 2019. Total number of patients in the study is 1,538 (TAVR 328, SAVR 1,210). Studies were done in the USA and Germany. The follow-up in studies lasted for a year or longer. All studies included studied outcome which is shown in Table 1 
Table 1. Demographic Profile of Included Studies

\begin{tabular}{lllllll}
\hline Studies & Year & Study type & TAVR age & SAVR Age & TAVR man & SAVR man \\
\hline Fox et al [17] & 2013 & Retrospective & $70 \pm 7$ & $67 \pm 8$ & 5 & 14 \\
Buttner et al [15] & 2016 & Retrospective & 69 & 65.5 & - & - \\
Herzog et al [18] & 2019 & Retrospective & 72.9 & 66.7 & 57 & 141 \\
Voudris et al [19] & 2019 & Retrospective & $70.5 \pm 8.5$ & $62.7 \pm 8.5$ & 155 & 641 \\
\hline
\end{tabular}

TAVR: transcutaneous aortic valve replacement; SAVR: surgical aortic valve replacement.

Table 2. New-Ottawa Scale for Quality Assessment of Studies

\begin{tabular}{llllll}
\hline Studies & Selection & Compatibility & Exposure & Total score & Adequate follow-up \\
\hline Fox et al [17] & $* * *$ & $* *$ & $* * *$ & 8 & $*$ \\
Buttner et al [15] & $* * *$ & $* *$ & $* * *$ & 8 & $*$ \\
Herzog et al [18] & $* * * *$ & $*$ & $* * *$ & 8 & $*$ \\
Voudris et al [19] & $* * * *$ & $* *$ & $* *$ & 9 & $\mathrm{xx}$ \\
\hline
\end{tabular}

$[15,17-19]$. Mean age of patients was more than 65 in both groups.

\section{Quality of study}

The overall quality of the included studies was moderate. All studies were observational and retrospective, posing some theoretical risk of selection bias due to incomplete randomization and inadequate allocation concealment. We used New-Ottawa Scale to access the quality and all studies scored $8-9$ on the scale of 1 - 9 (Table 2) [15, 17-19].

Since the research was a meta-analysis and there was no direct patient interaction, Institutional Review Board (IRB) Approval and Ethical Compliance With Human Study were not applicable.

\section{Results}

Study characteristics were almost similar between the two groups. The average age for TAVR was $69.45 \pm 8.27$ and for

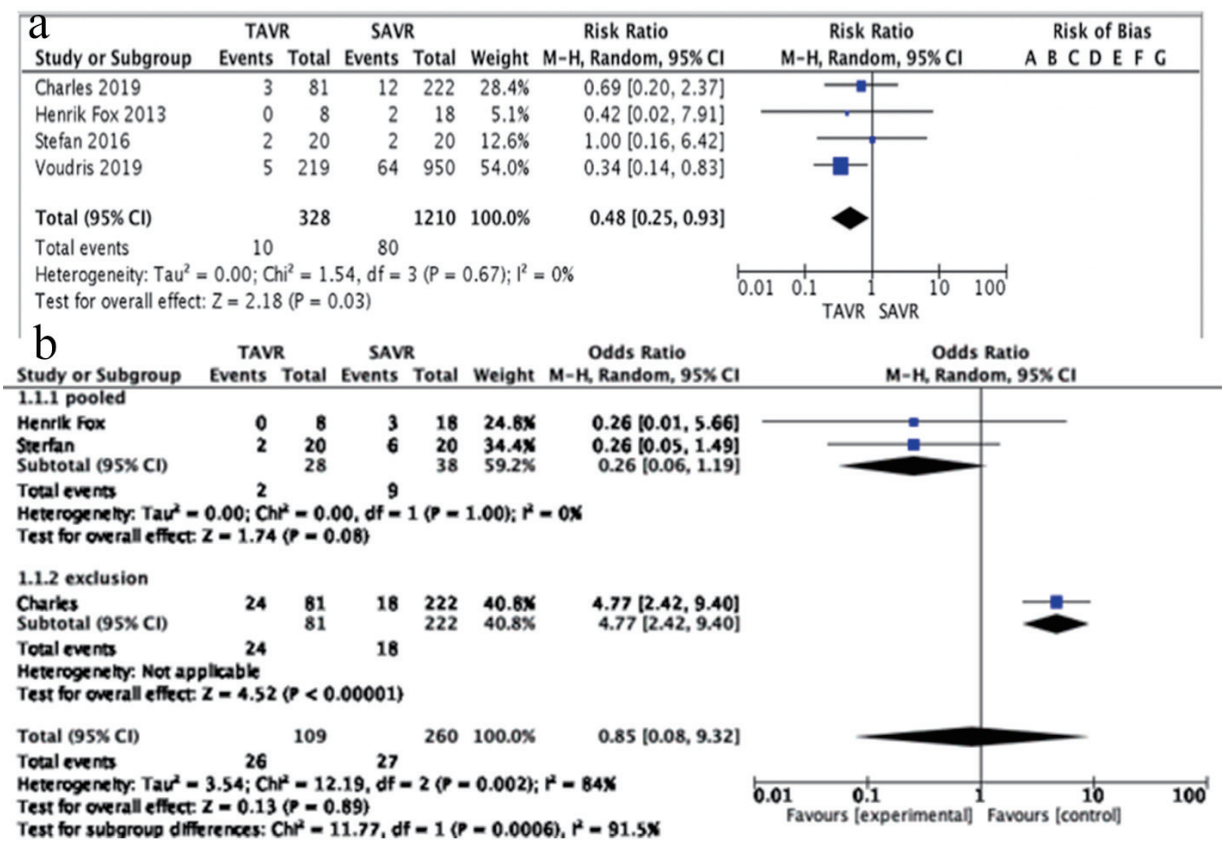

Figure 2. (a) 30-days mortality plot with OR and 95\% CI. TAVR has low mortality rates than SAVR. Results were statistically significant. (b) 1-year mortality. TAVR has comparable rates of 1-year mortality as SAVR. OR: odds ratio; Cl: confidence interval; TAVR: transcutaneous aortic valve replacement; SAVR: surgical aortic valve replacement. 


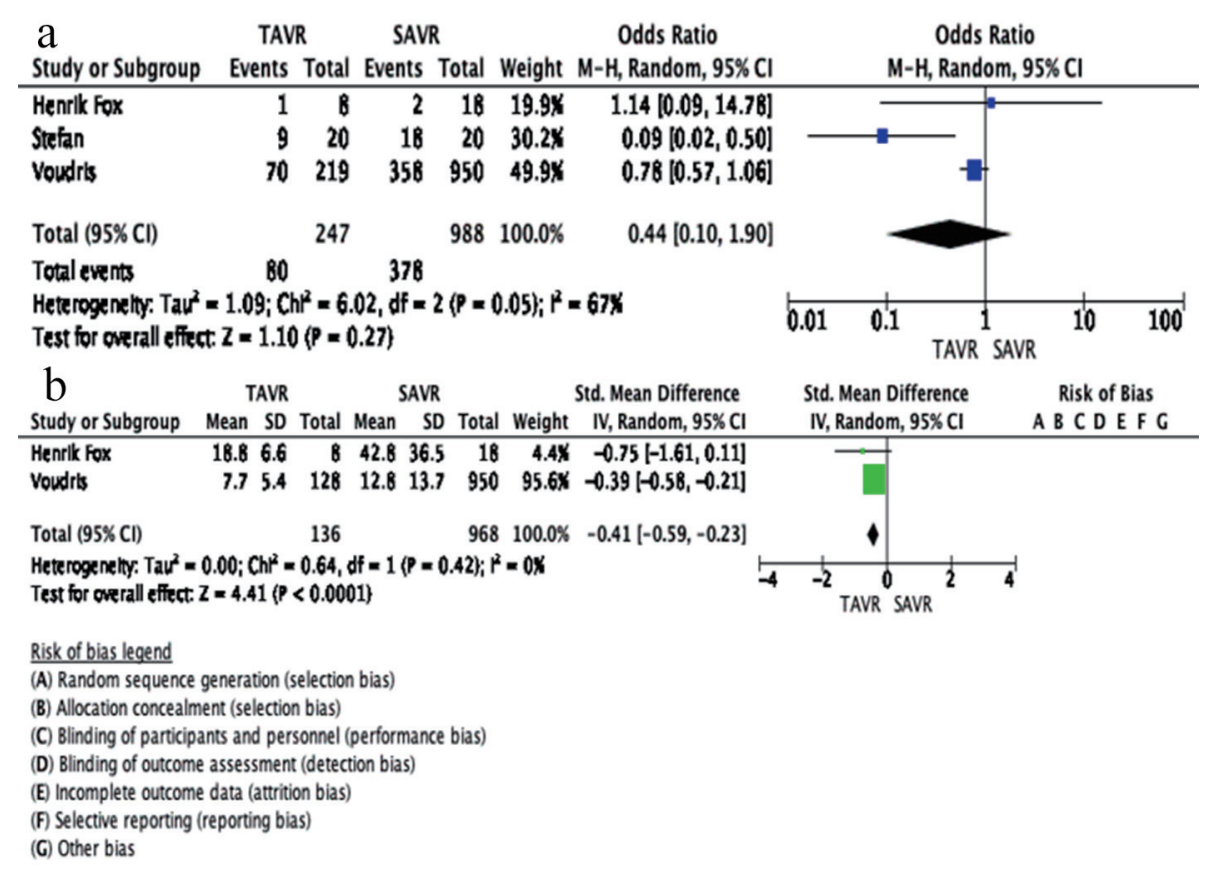

Figure 3. (a) AKI has comparable results in TAVR and SAVR. (b) Hospital stay, TAVR has better rates than SAVR. AKI: acute kidney injury; TAVR: transcutaneous aortic valve replacement; SAVR: surgical aortic valve replacement.

SAVR $64.68 \pm 11.29$. Majority of patients were men in both groups (TAVR 70.45\%, SAVR 66.89\%).

\section{Primary composite outcome (30-day/in-hospital mortality,} 1-year mortality, AKI)

The 30-day in-hospital mortality was studied in 1,538 patients (TAVR 328, SAVR 1,210). TAVR had better outcomes in terms of 30-day mortality than SAVR (odds ratio (OR): $0.48,95 \%$ CI: $0.25-0.93 ; \mathrm{P}=0.03$ ) (Fig. 2a). After the exclusion of Voudris et al, mortality at 30 day is comparable between the two groups (OR: 0.72, 95\% CI: $0.27-1.91 ; \mathrm{P}=0.51$ ) [19].

One-year mortality was studied in 369 patients (TAVR 109, SAVR 260). SAVR had comparable outcomes as TAVR in terms of 1-year mortality (OR: $0.76,95 \%$ CI: $0.10-5.51$; P $=0.78$ ). The results are mainly determined by Stefan et al in view of big sample size (Fig. 2b). AKI was studied in 1,235 patients (TAVR 247, SAVR 988). TAVR had comparable outcome as SAVR in terms of AKI (OR: 0.44, 95\% CI: 0.10 $1.90 ; \mathrm{P}=0.27$ ) (Fig. 3a). Hospital stay in days was studied in a total of 1,195 patients (TAVR 227, SAVR 968). TAVR has very low hospital stay in days as compared to SAVR (OR: 0.41, 95\% CI: -0.59 to -0.23 ; $\mathrm{P}<0.00001$ ) (Fig. 3 b).

\section{Publication bias}

The publication bias was illustrated graphically with funnel plotting. The vertical axis of the plot used standard error to estimate the sample size of the study, plotting large population studies on top and smaller at the bottom. The horizontal spread reflected the power and effect size of the included studies. On visual assessment, our funnel plot was symmetrical, indicating that the limited scatter was due to sampling variation and not publication bias (Fig. 4).

\section{Discussion}

Our meta-analysis is the first study to compare TAVR versus SAVR in kidney transplant patients. TAVR had better odds in terms of survival outcome at 30 days and hospital stay than SAVR in patients with kidney transplant. However, on longterm follow-up mortality results are comparable between the two procedures. TAVR and SAVR procedures have comparable AKI outcomes post procedure in kidney transplant patients.

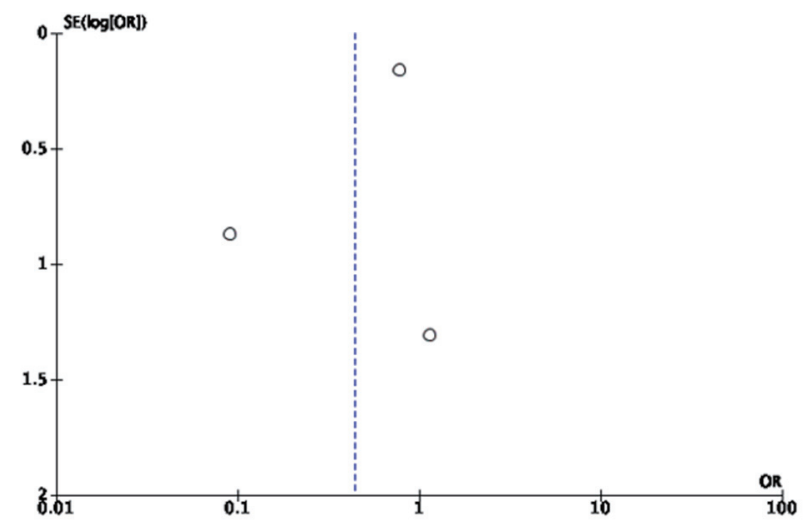

Figure 4. Funnel plot for bias of study. There is no significant scatter on the funnel plot which relates to less bias in publication. 
The duration of hospital stays post procedure had low odds for TAVR procedure than SAVR. Our overall results show that patients with kidney transplant are more inclined towards TAVR than SAVR.

Fox et al (2013) studied 26 patients for primary outcome in terms of survival (TAVR 8, SAVR 18); mortality at 30 days was $0 \%$ versus $11.1 \%$ and 1 -year mortality was $0 \%$ versus $16.7 \%$ in TAVR versus SAVR respectively. AKI was reported in $12.5 \%$ versus $11.11 \%$ in TAVR versus SAVR. Comparison of hospital stay was not significant between the groups $(\mathrm{P}=$ 0.084) [17]. Buttner et al (2016) studied a total 40 of patients (TAVR 20 vs. SAVR 20) for primary outcome of mortality and AKI; 30-day mortality was $10 \%$ versus $10 \%$ and 1 -year mortality was $10 \%$ versus $30 \%$ in TAVR versus SAVR [15]. Herzog et al (2019) studied 303 patients; 30-day mortality was $3.7 \%$ versus $5.4 \%$ in TAVR versus SAVR respectively. The final results of our study for 1-year mortality were more determined by this study $(30.2 \%$ vs. $12.4 \%)$ [18]. Voudris et al (2019) recently studied 1,167 patients for complications post procedure; 30-day mortality was $2.3 \%$ versus $6.7 \%$ with $\mathrm{P}=$ 0.032 . AKI results were comparable $(31.96 \%$ vs. $37.68 \%$; $\mathrm{P}$ $=0.120$ ) [19]. The major limitations of all these studies were inclusion of the smaller scale population and being relatively underpowered to assess the long-term outcomes of the procedures. Overall, the findings of these studies should be interpreted with caution as they were subject to the biases inherent to the non-randomized assignments. This, along with the fact that all studies reported conflicting findings, motivated us to perform this meta-analysis.

Our study is the first to pool the findings from four studies including 1,538 patients (TAVR 328, SAVR 1,210). Our study revealed overall better odds for 30-day mortality with TAVR than SAVR. One-year mortality was comparable and results were determined by Herzog et al, as its total contribution was $82.12 \%$ (heterogeneity of results was high, $84 \%$ ) [18].

We compared our results with literature available. Moat et al have given a 30-day mortality following TAVR in high surgical risk patients of 7.1\% [20]. Wenaweser et al reported similar mortality rates in TAVR and SAVR at 30 days in high surgical risk patients [21]. Sharma et al reviewed data of 1,335 kidney transplant patients. Surgical valve replacement procedures are at higher risk of mortality in kidney transplant patients with an approximate rate of $20 \%$ per year [22]. Conradi et al in their study reported 30-day mortality in TAVR versus SAVR of 7.3\% and $8.6 \%$, however, without statistical difference [23]. Oneyear mortality was comparable in our study in TAVR group and SAVR group. A study by Ben-Dor et al revealed TAVR having higher mortality in patients with higher surgical risk than SAVR and balloon/medical management having worst outcome [24]. Wenaweser et al have reported 1-year mortality of $18.7 \%$ for SAVR and $17.1 \%$ for high surgical risk patients [21]. Conradi et al have reported better results trending towards SAVR than TAVR in high surgical risk patients [23].

Our study revealed TAVR and SAVR in kidney transplant with comparable rates of AKI. Bagur et al revealed a higher incidence of AKI in patients who had SAVR than TAVR [25].

Our study revealed that TAVR is associated with low hospital days of admission than SAVR in kidney transplant patients. Conradi et al have reported more days of hospitalization in TAVR than SAVR in high risk surgical patients [23].

\section{Limitations}

Our study is constrained by the limitations of the included studies. Three studies were abstracts where we did not have exact details of comorbidities; however, studies have mentioned that baseline comorbidity was almost similar. A significant barrier was our inability to perform a stratified subgroup analysis based on the variable follow-up durations and different selection criteria. The inherent heterogeneity in the observational data limits our ability to draw definitive conclusions about the outcomes in kidney transplant patient undergoing TAVR versus SAVR. The predictive odds of all the components could not be calculated due to insufficient reporting of the stratified event rates.

\section{Conclusions}

Our study suggests that in patients with a history of kidney transplant, both SAVR and TAVR have a comparable risk of $\mathrm{AKI}$ and mortality at both short- and long-term follow-up.

\section{Acknowledgments}

None to declare.

\section{Financial Disclosure}

None to declare.

\section{Conflict of Interest}

None to declare.

\section{Informed Consent}

Not applicable.

\section{Author Contributions}

FD coordinated the data collection; WU and KZ did the statistical analysis and helped in manuscript; TM and MCA wrote the manuscript; YS helped in reference arrangement and data mining; ZH and HMP helped in literature review and data collection; LF helped in manuscript; and DG and JJG did the critical review.

\section{Data Availability}

Any inquiries regarding supporting data availability of this 
study should be directed to the corresponding author.

\section{References}

1. Ahmad T, Kishore KS, Maheshwarappa NN, Pasarad AK. Challenges of valve surgeries in post-renal transplant patients. Ann Card Anaesth. 2015;18(4):603-605.

2. Sarnak MJ. Cardiovascular complications in chronic kidney disease. Am J Kidney Dis. 2003;41(5 Suppl):11-17.

3. Du X, Soon JL. Mild to moderate aortic stenosis and coronary bypass surgery. J Cardiol. 2011;57(1):31-35.

4. Messika-Zeitoun D, Lloyd G. Aortic valve stenosis: evaluation and management of patients with discordant grading.

5. London GM, Pannier B, Marchais SJ, Guerin AP. Calcification of the aortic valve in the dialyzed patient. J Am Soc Nephrol. 2000;11(4):778-783.

6. Umana E, Ahmed W, Alpert MA. Valvular and perivalvular abnormalities in end-stage renal disease. Am J Med Sci. 2003;325(4):237-242.

7. Thourani VH, Keeling WB, Sarin EL, Guyton RA, Kilgo PD, Dara AB, Puskas JD, et al. Impact of preoperative renal dysfunction on long-term survival for patients undergoing aortic valve replacement. Ann Thorac Surg. 2011;91(6):1798-1806; discussion 1806-1797.

8. Ifedili IA, Bolorunduro O, Bob-Manuel T, Heckle MR, Christian E, Kar S, Ibebuogu UN. Impact of Pre-existing Kidney Dysfunction on Outcomes Following Transcatheter Aortic Valve Replacement. Curr Cardiol Rev. 2017;13(4):283-292.

9. Edwards FH, Peterson ED, Coombs LP, DeLong ER, Jamieson WR, Shroyer ALW, Grover FL. Prediction of operative mortality after valve replacement surgery. J Am Coll Cardiol. 2001;37(3):885-892.

10. Horst M, Mehlhorn U, Hoerstrup SP, Suedkamp M, de Vivie ER. Cardiac surgery in patients with end-stage renal disease: 10-year experience. Ann Thorac Surg. 2000;69(1):96-101.

11. Leon MB, Smith CR, Mack M, Miller DC, Moses JW, Svensson LG, Tuzcu EM, et al. Transcatheter aortic-valve implantation for aortic stenosis in patients who cannot undergo surgery. N Engl J Med. 2010;363(17):1597-1607.

12. Mack MJ, Leon MB, Thourani VH, Makkar R, Kodali SK, Russo M, Kapadia SR, et al. Transcatheter aorticvalve replacement with a balloon-expandable valve in low-risk patients. N Engl J Med. 2019;380(18):16951705.

13. Popma JJ, Deeb GM, Yakubov SJ, Mumtaz M, Gada H, O'Hair D, Bajwa T, et al. Transcatheter aortic-valve replacement with a self-expanding valve in low-risk patients. N Engl J Med. 2019;380(18):1706-1715.

14. Szerlip M, Zajarias A, Vemalapalli S, Brennan M, Dai D, Maniar H, Lindman BR, et al. Transcatheter aortic valve replacement in patients with end-stage renal disease.
J Am Coll Cardiol. 2019;73(22):2806-2815.

15. Buttner S, Weiler H, Zoller C, et al. TCT-677 Comparison of surgical versus interventional treatment of aortic valve stenosis in kidney transplant recipients. Journal of the American College of Cardiology. 2016;68:B274.

16. Szerlip M, Kim RJ, Adeniyi T, Thourani V, Babaliaros V, Bavaria J, Herrmann HC, et al. The outcomes of transcatheter aortic valve replacement in a cohort of patients with end-stage renal disease. Catheter Cardiovasc Interv. 2016;87(7):1314-1321.

17. Fox H, Buttner S, Hemmann K, Asbe-Vollkopf A, Doss M, Beiras-Fernandez A, Moritz A, et al. Transcatheter aortic valve implantation improves outcome compared to open-heart surgery in kidney transplant recipients requiring aortic valve replacement. J Cardiol. 2013;61(6):423427.

18. Herzog CA, Wetmore JB, Gilbertson DT. Transcatheter vs. surgical aortic valve replacement in US renal transplant patients. Circulation. 2019;140:A12331-A12331.

19. Voudris K, Kavinsky C. TCT-709 aortic valve replacement in patients with prior kidney transplantation: transcatheter or surgical approach? Journal of the American College of Cardiology. 2019;74:B696.

20. Moat NE, Ludman P, de Belder MA, Bridgewater B, Cunningham AD, Young $\mathrm{CP}$, Thomas $\mathrm{M}$, et al. Longterm outcomes after transcatheter aortic valve implantation in high-risk patients with severe aortic stenosis: the U.K. TAVI (United Kingdom Transcatheter Aortic Valve Implantation) Registry. J Am Coll Cardiol. 2011;58(20):2130-2138.

21. Wenaweser P, Pilgrim T, Kadner A, Huber C, Stortecky S, Buellesfeld L, Khattab AA, et al. Clinical outcomes of patients with severe aortic stenosis at increased surgical risk according to treatment modality. J Am Coll Cardiol. 2011;58(21):2151-2162.

22. Sharma A, Gilbertson DT, Herzog CA. Survival of kidney transplantation patients in the United States after cardiac valve replacement. Circulation. 2010;121(25):27332739.

23. Conradi L, Seiffert M, Treede H, Silaschi M, Baldus S, Schirmer J, Kersten JF, et al. Transcatheter aortic valve implantation versus surgical aortic valve replacement: a propensity score analysis in patients at high surgical risk. J Thorac Cardiovasc Surg. 2012;143(1):64-71.

24. Ben-Dor I, Dvir D, Barbash IM, Okubagzi P, Torguson R, Xue Z, Lindsay J, et al. Outcomes of patients with severe aortic stenosis at high surgical risk evaluated in a trial of transcatheter aortic valve implantation. Am J Cardiol. 2012;110(7):1008-1014.

25. Bagur R, Webb JG, Nietlispach F, Dumont E, De Larochelliere R, Doyle D, Masson JB, et al. Acute kidney injury following transcatheter aortic valve implantation: predictive factors, prognostic value, and comparison with surgical aortic valve replacement. Eur Heart J. 2010;31(7):865-874. 